\title{
ANÁLISE DE VIABILIDADE ECONÔMICO-FINANCEIRA DE UM INVESTIMENTO EM UMA LOJA DE BRINQUEDOS INFANTIS
}

\author{
Miréia Torres Lima - mireiatorres1313@gmail.com \\ UNIVERSIDADE FEDERAL DO CEARÁ - UFC \\ Brunna dos Santos Brandão - brandao.brunna.santos@gmail.com \\ UNIVERSIDADE FEDERAL DO CEARÁ - UFC \\ Luiza Eduarda Aguiar Silveira - luizaeduardaas@gmail.com \\ UNIVERSIDADE FEDERAL DO CEARÁ - UFC \\ Beatriz Bezerra Barbosa - beatrizbrbs98@gmail.com \\ UNIVERSIDADE FEDERAL DO CEARÁ - UFC
}

\begin{abstract}
Resumo: Diante da busca constante por investimentos que proporcionem grandes retornos e da escassez dos recursos em época de crise econômica, faz-se necessário o uso de técnicas matemáticas e estatísticas para auxiliarem mesmo os pequenos empreendedores na análise econômico-financeira de seus empreendimentos. Neste contex to o presente artigo teve o objetivo de realizar um estudo de viabilidade econômicofinanceira da implantação de duas novas linhas de brinquedos em uma loja, tomando como referência outras duas linhas já existentes que correspondem aos produtos mais importantes, portanto pertencentes a curva A da loja, tal conclusão foi obtida a partir do conceito da curva $\mathrm{ABC}$. Durante o estudo foram coletadas informações relacionadas à movimentações financeiras, para a montagem do fluxo de caixa, e a partir dele foi possível utilizar métodos de modelagem e análises de investimentos, tais como o Valor Presente Líquido (VPL), a Taxa Interna de Retorno (TIR) e o Ponto de Fischer caso necessário, para concluir se o novo investimento era viável ou não. A metodologia utilizada é de natureza descritiva e exploratória, pois foi possível a partir do fornecimento de informações da proprietária e explora o máximo de informações do objeto de estudo. Como resultados observou-se que o investimento não pode ser considerado viável, visto que o mesmo não apresentou valor superior ao das linhas já existentes, sendo, desta forma, preferível continuar apenas com as duas linhas antigas ou procurar novas formas de investimento.
\end{abstract}

Palavras-chaves: Análise de viabilidade econômico-financeira, Fluxo de Caixa, microempreendimento.

Abstract: In view of the constant search for investments that provides great returns and scarcity of resources in periods of economic crisis, it is necessary to use mathematical and statistical techniques to assist the same entrepreneurs in the economic and financial 
analysis of their enterprises. In this context, this article aimed to carry out an economicfinancial feasibility study on the implementation of two new toy lines in a store, using as reference two other existing lines, which are applied to the most important products, therefore, the A curve of the store, this conclusion was initiated in the ABC curve concept. During the study, information related to financial movements was collected, to assemble cash flow, and from it it was possible to use modeling methods and investment statistics, such as Net Present Value (NPV), an Internal Rate of Return (IRR) and Fischer's Point needed to conclude whether the new investment was viable or not. The methodology used is descriptive and exploratory in nature, as it was possible to start with the provision of proprietary information and explore as much information as possible about the object of study. Like the approved results, if the investment cannot be considered viable, since it does not, show a higher value than the existing lines, being, therefore, preferable to continue with only two old lines or to look for new forms of investment.

Keywords: Economic-financial feasibility analysis, Cash flow, micro-enterprise.

\section{Introdução}

No atual contexto de crise econômica em que o país se insere o empreendedorismo de necessidade surge como uma solução para driblar o desemprego. Com isso cerca de 11,1 milhões de empresas foram criadas no Brasil nos últimos 3,5 anos e em 2011 as micro e pequenas empresas movimentaram 27\% do Produto Interno Bruto (PIB) nacional (SEBRAE, 2014).

Devido à importância desses empreendimentos para economia nacional, a sustentabilidade econômica deles que muitas vezes é comprometida pelo despreparo e falta de conhecimento técnico, é um fator crucial, pois são responsáveis por mais da metade dos empregos formais criados e apresentam-se como grandes geradores de riqueza. Com disso deve-se mostrar à esses empreendedores a possibilidade de utilizar técnicas matemáticas e estatísticas para fazer uma análise econômico-financeira de seus empreendimentos (SAMANEZ, 2009) e com isso aumentar possibilidade de permanência no mercado.

Diante desse contexto o presente artigo tem o objetivo de apresentar um estudo analítico que visa examinar a viabilidade econômico-financeira de um investimento em uma loja de brinquedos infantis no estado do Ceará, classificada como micro empreendimento. Este artigo é constituído a partir de uma fundamentação teórica que aborda conceitos e definições ligadas a análise de investimentos, sobretudo de métodos como o VPL (Valor 
Presente Líquido) e a TIR (Taxa Interna de Retorno), destacando a possibilidade de utilizar técnicas complementares como o Ponto de Fischer caso esses dois métodos não sejam suficientes para concluir a análise, e a importância da elaboração do FCL (Fluxo de Caixa Livre) no fornecimento das informações. Em seguida, apresenta-se a metodologia utilizada, o estudo de viabilidade econômico-financeiro do investimento da loja sob análise, além da discussão dos resultados obtidos, e finalmente são expostas as conclusões dos autores, as limitações do estudo e as recomendações para trabalhos futuros.

\section{Fundamentação teórica}

\subsection{Análise de investimentos}

A realização de um investimento numa empresa pode ser a contratação de um novo funcionário, a decisão de terceirizar determinado serviço ou realizá-lo por conta própria, adquirir ou substituir equipamentos, entre outros, podem levá-la a desembolsar capital, ou seja, comprometer capital no presente para obter um retorno positivo no futuro. Tendo em vista a relevância dessas ações para a organização, pois representam desembolso de capital, faz-se necessário a análise e seleção desses investimentos da maneira mais assertiva possível (MARTINS, 1990; GITMAN, 2010).

Um elemento crucial para assegurar a confiabilidade dessa análise é o Fluxo de Caixa Livre (FCL), sobretudo deve-se realizar seus cálculos e projeções corretamente para as possíveis alternativas de investimento. Ele consiste na descrição de todas as entradas e saídas de capital ao longo da vida útil do projeto em análise, levando em consideração o valor do dinheiro no tempo e o custo de oportunidade. A partir dessas informações é possível aplicar as ferramentas de análise de investimentos e tomar decisão a respeito da adoção da alternativa mais vantajosa do ponto de vista econômico-financeiro (ASSAF NETO, 2005).

Segundo Samanez (2009) o FCL mais básico é constituído preferivelmente pelo fluxo positivo gerado pela receita bruta com deduções menos os custos e despesas necessários para assegurar a continuidade e desenvolvimento do projeto, e menos a mudança de capital de giro. A depreciação de ativos e outras despesas "não-caixa", devem ser somadas novamente depois de ser considerado seu efeito fiscal. 
No quadro 1 está apresentado um modelo de FCL que possui informações a respeito das receitas, custos e despesas (fixas e variáveis), impostos, benefícios fiscais, investimentos, injeção e recuperação de capital de giro e valor residual do ativo em caso de venda.

Quadro 1 - Modelo de Fluxo de Caixa Livre

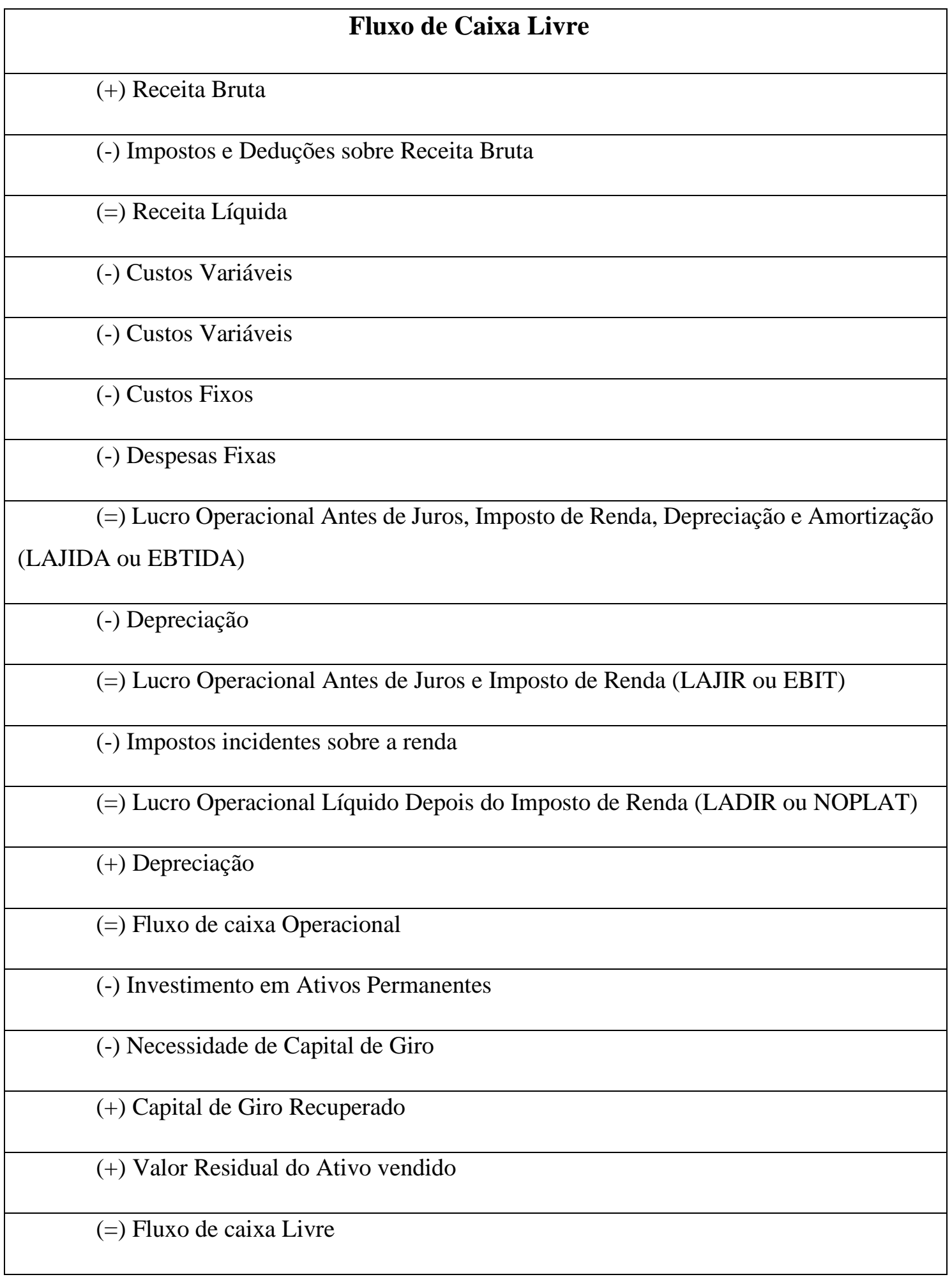

Fonte: Adaptado de Bruni(2008) e Samanez (2009) 
Ressaltar que os FCLs produzidos em cada alternativa de investimento do projeto serão utilizados para análise de viabilidade econômico-financeira descrita a seguir, usando os métodos VPL e TIR.

\subsection{Métodos de análise de viabilidade econômico-financeira de investimentos}

\subsubsection{Valor Presente Líquido (VPL)}

De acordo com Bruni e Famá (2012) o VPL, é constituído do somatório na data zero dos fluxos de caixa futuro. Esse método avalia se o investimento é vantajoso ou não, sendo calculado pela seguinte fórmula:

$$
V P L=-I+\sum_{t=1}^{n} \frac{F C t}{(1+j)^{t}}
$$

Equação 1 - Fórmula do VPL

Sendo:

- FCt: fluxo de caixa no t-ésimo período;

- I: investimento inicial;

- K: custo do capital ou taxa mínima de atratividade;

- t: tempo de desconto de cada entrada de caixa;

- n: tempo de desconto do último fluxo de caixa;

A aplicação para tomada de decisão a respeito do investimento analisado é: se o VPL seja maior que zero o projeto é considerado viável, pois supera o investimento inicial, caso contrário o projeto é considerado economicamente inviável, e comparando dois investimentos aquele que apresentar maior VPL, será o mais vantajoso.

\subsubsection{Taxa Interna de Retorno (TIR)}

A TIR busca encontrar uma taxa intrínseca de rendimento. Sua definição é a taxa de retorno do investimento (SAMANEZ, 2009). Através da fórmula a seguir, conclui-se que a TIR é uma taxa fictícia que zera o VPL: 


$$
V P L=-I+\sum_{t=1}^{n} \frac{F C t}{(1+i *)^{t}}=0
$$

Equação 2 - Fórmula do TIR

O critério de análise é que se a TIR for maior que a Taxa Mínima de Atratividade (TMA), o investimento pode ser considerado economicamente viável, e comparando dois investimentos aquele que apresentar maior TIR será o mais vantajoso.

\subsubsection{Ponto de Fisher}

O ponto de Fisher é uma medida para se analisar um investimento, ele é capaz de analisar a dimensão de risco do mesmo (Souza e Clemente, 2004). O ponto de Fisher analisa o momento em que o projeto possui uma melhor viabilidade de investimento comparado a outro investimento, ou seja, quando a taxa de rentabilidade de Fisher passa a ser melhor do que a taxa mínima de atratividade.

Para Kassai (1996), a análise que estabelece a relação entre a Taxa Interna de Retorno (TIR) e o Valor Presente Líquido (VPL) é que indica o chamado ponto de intersecção de Fischer.

\section{Metodologia}

A pesquisa tratou-se de um estudo de caso de objetivo descritivo e modalidade exploratória. A coleta de dados foi realizada a partir das informações das movimentações financeiras fornecidas pela proprietária acerca de duas linhas de brinquedos que retornavam maior lucro à loja, para que então pudesse ser feita uma comparação com as duas linhas que ela tinha interesse de investir. Quanto a modalidade estabeleceu-se o objetivo de identificar se o novo investimento seria vantajoso, e a partir disso explorouse todas as informações necessárias para alcançá-lo.

Os dados coletados de cada linha de brinquedo em questão para a montagem do FCL e posterior análise foram: os custos de compra unitária, o valor de venda aplicado e o custo de venda total. Em seguida, o processo de análise prosseguiu da seguinte forma:

- Estabeleceu-se um horizonte de análise de três meses, tomando como data zero o mês de outubro;

- Montou-se um fluxo de caixa para esse horizonte;

-E aplicou-se os métodos de análise de viabilidade econômico-financeira do investimento, sendo eles VPL, TIR e Ponto de Fischer. 
Vale salientar que esses dados só foram obtidos através do acesso da planilha financeira da loja com todas as informações a respeito dos custos, despesas e receitas do antigo investimento. Desse modo, em posse dessas informações foi possível realizar o estudo de maneira completa e confiável.

\section{Análise de viabilidade econômico-financeira do investimento}

A empresa em estudo possui como atividade a venda de brinquedos infantis com foco naqueles de cunho educacional, que auxiliam no desenvolvimento cognitivo da criança. Buscando elevar o faturamento, a empresa mencionada estuda a implantação de duas novas linhas de brinquedo, usando como comparativo duas linhas que já são vendidas. Desse modo, o objeto de estudo é o investimento na renovação das linhas de brinquedos vendidos.

\subsection{Valor do Investimento e Taxa de Atratividade}

O valor estimado para o investimento na linha de brinquedos foi feito com base na pesquisa de preços do mercado e foi correspondente a $\mathrm{R} \$ 13.547,86$. Por meio de entrevista com a proprietária, a taxa de atratividade estabelecida foi de $5 \%$.

\subsection{Fluxo de caixa livre do investimento}

A partir da obtenção dos dados: receitas líquidas projetadas, despesas administrativas e financeiras, depreciação, taxa do imposto de renda e investimento líquido em equipamento, tornou-se possível a elaboração do fluxo de caixa livre do investimento em questão. Para sua construção, não levou-se em consideração custos fixos e variáveis, somente o Custo do Produto Vendido (CPV) para facilitar os cálculos, nele o custo total de brinquedos das linhas de investimento comprados se caracteriza como o estoque que a empresa teria ao longo do mês, possuindo apenas gastos de armazenamento até serem vendidos.

Para a elaboração da análise de investimento, foi realizada uma projeção de três meses com início no mês de novembro, para o atual e o novo investimento, baseando-se nas receitas do primeiro para concluir a respeito da viabilidade ou não do investimento. A seguir estão apresentados os FCLs produzidos e os valores do VPL e da TIR de ambos investimentos:

Quadro 2 - FCL para o atual investimento no horizonte de análise estabelecido. 
INVESTIMENTO ATUAL

\begin{tabular}{|c|c|c|c|c|c|c|}
\hline & & 0 & Nov & & Dez & Jan \\
\hline (+) Receitas & & & $\mathrm{R} \$ 30.059,34$ & $\mathrm{R} \$$ & $32.764,68$ & $\mathrm{R} \$ 35.713,50$ \\
\hline (-) CPV & & & $-\mathrm{R} \$ 15.029,67$ & $-\mathrm{R} \$$ & $16.382,34$ & $-\mathrm{R} \$ 17.856,75$ \\
\hline (-) Deprec & & & 250,49 & $-\mathrm{R} \$$ & 250,49 & 250,49 \\
\hline (=) LLAIR & & & $\mathrm{R} \$ 14.779,18$ & $\mathrm{R} \$$ & $16.131,85$ & $\mathrm{R} \$ 17.606,26$ \\
\hline$(-)$ IR & & & $-\mathrm{R} \$ 2.660,25$ & & $-\mathrm{R} \$ 2.903,73$ & $-\mathrm{R} \$ 3.169,13$ \\
\hline (=) LLOpDIR & & & $\mathrm{R} \$ 12.118,92$ & $\mathrm{R} \$$ & $13.228,11$ & $\mathrm{R} \$ 14.437,13$ \\
\hline (+) Deprec. & & & 250,49 & $\mathrm{R} \$$ & 250,49 & 250,49 \\
\hline (=) FCOp & & & $\mathrm{R} \$ 12.369,42$ & $\mathrm{R} \$$ & $13.478,61$ & $\mathrm{R} \$ 14.687,62$ \\
\hline$(+/-)$ Investim./desinv. líq.em equip. & & $-\mathrm{R} \$ 15.029,67$ & & & & \\
\hline \multicolumn{7}{|l|}{$(+/-)$ Investim./desinv. em cap. de giro } \\
\hline \multirow[t]{7}{*}{ (=) FCLivre } & & $-\mathrm{R} \$ 15.029,67$ & $\mathrm{R} \$ 12.369,42$ & $\mathrm{R} \$$ & $13.478,61$ & $\mathrm{R} \$ 14.687,62$ \\
\hline & & EC & Saldo & VP & & FCD \\
\hline & 0,00 & $-15.029,67$ & 0,00 & & 0,00 & 0,00 \\
\hline & 1,00 & $12.369,42$ & $-2.660,25$ & & $11.780,40$ & $-3.249,27$ \\
\hline & 2,00 & $13.478,61$ & $10.818,36$ & & $12.225,49$ & $8.976,22$ \\
\hline & 3,00 & $14.687,62$ & $25.505,98$ & & $12.687,72$ & $21.663,95$ \\
\hline & & Payback Simples & 1,20 & Paybc & ack Descontadc & 1,27 \\
\hline TIR & & $69 \%$ & & & & \\
\hline VPL & & $\mathrm{R} \$ 21.663,95$ & & & & \\
\hline
\end{tabular}

Fonte: Autores

Quadro 3 - FCL para o novo investimento no horizonte de análise estabelecido. 
NOVO INVESTIMENTO

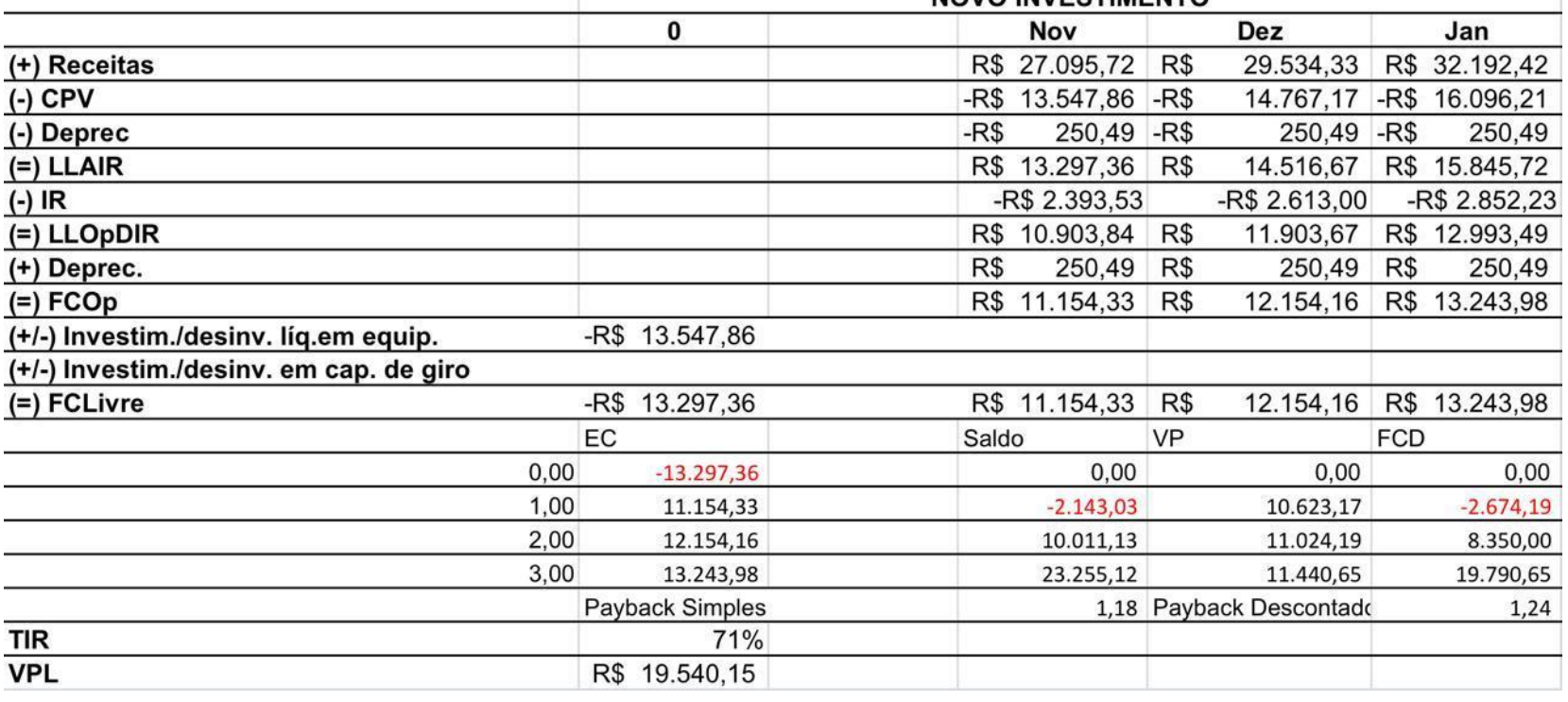

Fonte: Autores

\subsection{Cálculo do VPL e da TIR}

Os cálculos da TIR e do VPL para o atual e novo investimento foram feitos pelo Software Microsoft Excel, porém as demonstrações matemáticas de ambos podem ser expressas respectivamente da seguinte forma:

$$
\begin{gathered}
15029,67=\frac{12369,42}{1+i^{1}}+\frac{13478,61}{1+i^{2}}+\frac{14687,62}{1+i^{3}} \quad \text { TIR atual }=69 \% \\
13547,86=\frac{11154,33}{1+i^{1}}+\frac{12154,16}{1+i^{2}}+\frac{13243,98}{1+i^{3}} \quad \text { TIR novo }=71 \% \\
V P L=\frac{12369,42}{1+0,05^{1}}+\frac{13478,61}{1+0,05^{2}}+\frac{14687,62}{1+0,05^{3}} \quad \text { VPL atual }=\mathrm{R} \$ 21663,95 \\
V P L=\frac{11154,33}{1+0,05^{1}}+\frac{12154,16}{1+0,05^{2}}+\frac{13243,98}{1+0,05^{3}} \quad \text { VPL novo }=\mathrm{R} \$ 19540,15
\end{gathered}
$$

Observa-se que com os dados lançados nas fórmulas de cálculo do VPL e da TIR, a partir do software Microsoft Excel, obteve-se os mesmos resultados.

Através desses resultados foi possível concluir que os dois projetos são viáveis economicamente. A tabela 1 apresenta a comparação entre o VPL e a TIR dos dois investimentos, ao analisá-la identificou-se uma ambiguidade na decisão de qual investimento escolher, pois pelo critério do VPL deve-se escolher a atual linha de brinquedos, já que apresenta maior valor, já pela TIR deve-se escolher o novo 
investimento pois gera um maior retorno. Diante disso para embasar a decisão utilizouse o Ponto de Fischer.

Tabela 1 - Comparação da TIR e do VPL entre o atual e o novo investimento.

\begin{tabular}{|c|c|c|}
\hline & Atual investimento & Novo investimento \\
\hline VPL & $21.663,95$ & $19.540,15$ \\
\hline TIR & $69 \%$ & $71 \%$ \\
\hline
\end{tabular}

Fonte: Autores

\subsection{Cálculo do Ponto de Fisher}

Para a análise desse ponto é necessário montar um fluxo de caixa incremental, que consiste no fluxo resultante da operação de subtração do atual e do o novo investimento. A tabela 2 apresenta os fluxos de caixa projetados e o incremental de ambos investimentos.

Tabela 2 -Fluxo de Caixa projetado dos investimentos atual e novo, e incremental .

\begin{tabular}{|c|c|c|c|}
\hline \multicolumn{4}{|c|}{ Fluxos de caixas da análise } \\
\hline Mês & $\begin{array}{c}\text { Atual } \\
\text { investimento }\end{array}$ & $\begin{array}{c}\text { Novo } \\
\text { investimento }\end{array}$ & Atual - Novo \\
\hline Outubro & $-15.029,67$ & $-13.297,36$ & $-1.732,31$ \\
\hline Novembro & $12.369,42$ & $11.154,33$ & $1.215,09$ \\
\hline Dezembro & $13.478,61$ & $12.154,16$ & $1.324,44$ \\
\hline Janeiro & $14.687,62$ & $13.243,98$ & $1.443,64$ \\
\hline
\end{tabular}

Fonte: Autores

Em seguida foram estabelecidos os valores da TIR e do VPL do fluxo de caixa incremental que correspondem respectivamente a $55 \%$ e $\mathrm{R} \$ 2.600,99$, e de pontos estratégicos dos fluxos de caixa dos investimentos como o valor do VPL nas taxas de 0\% que correspondem ao instante inicial, na TMA estabelecida pela proprietária, na TMA de Fischer encontrada a partir do fluxo de caixa incremental e na TIR de cada investimento. Tais informações estão dispostas nas tabelas 3 e 4 . Foram utilizadas para a confecção do gráfico 1 que proporcionou uma melhor visualização da situação.

Tabela 3 - Pontos importantes do atual investimento

\begin{tabular}{|c|c|}
\hline \multicolumn{2}{|c|}{ Atual Investimento } \\
\hline Taxa interna & VPL \\
\hline $0 \%$ & $\mathrm{R} \$ 25.505,98$ \\
\hline $5 \%$ & $\mathrm{R} \$ 21.663,95$ \\
\hline $55 \%$ & $\mathrm{R} \$ 2.600,99$ \\
\hline $69 \%$ & $\mathrm{R} \$ 0,00$ \\
\hline
\end{tabular}

Fonte: Autores

Tabela 4 - Pontos importantes do novo investimento 


\begin{tabular}{|c|c|}
\hline \multicolumn{2}{|c|}{ Novo Investimento } \\
\hline Taxa interna & VPL \\
\hline $0 \%$ & $\mathrm{R} \$ 23.255,12$ \\
\hline $5 \%$ & $\mathrm{R} \$ 19.540,15$ \\
\hline $55 \%$ & $\mathrm{R} \$ 2.600,99$ \\
\hline $71 \%$ & $\mathrm{R} \$ 0,00$ \\
\hline \multicolumn{2}{|c|}{ Fonte: Autores }
\end{tabular}

Figura 1 - Curva do VPL pelo TIR

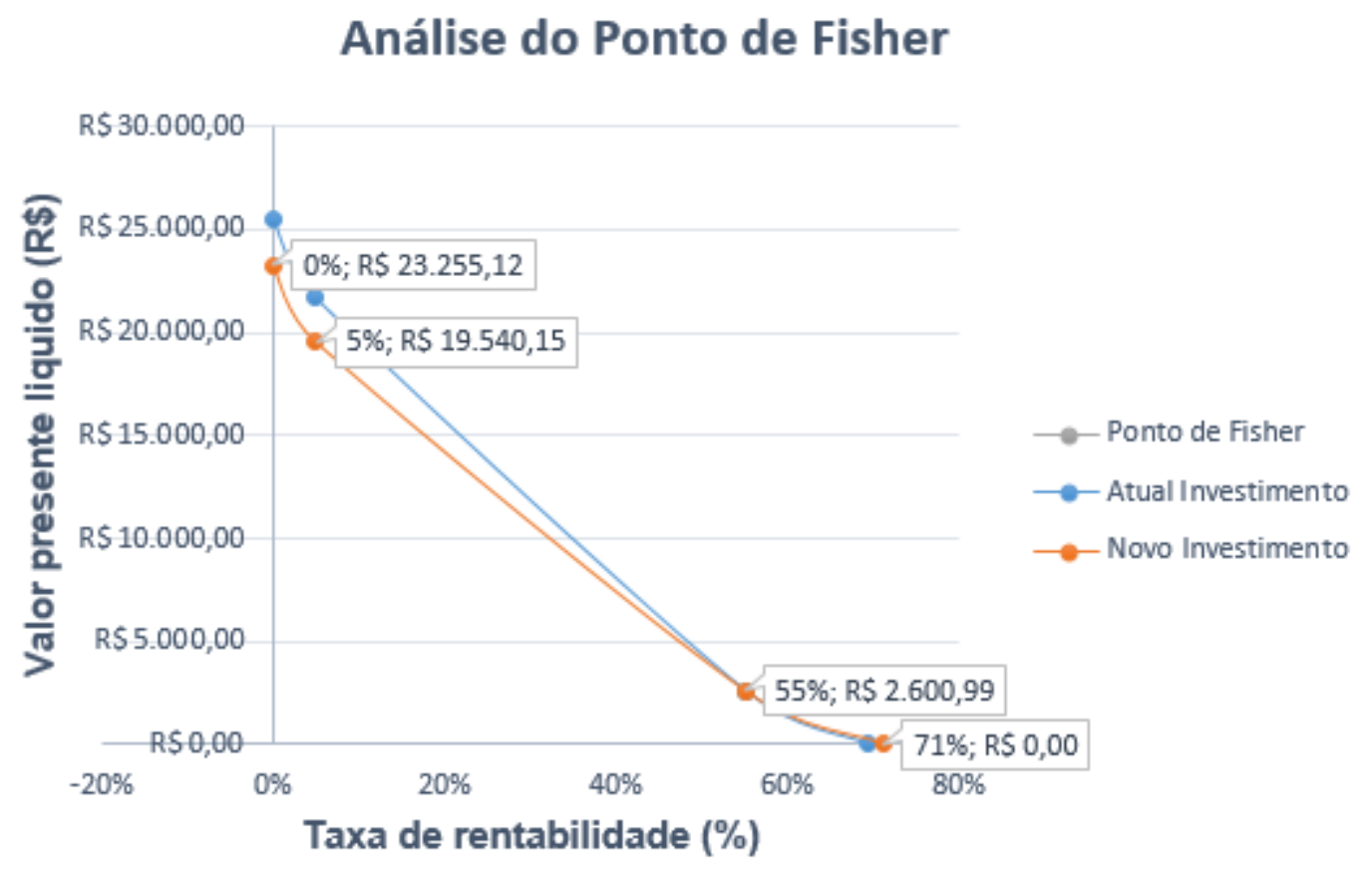

Fonte: Autores

\subsubsection{Análise do VPL em relação à TMA}

- O ponto de equilíbrio entre os fluxos do atual e novo investimento, que corresponde ao Ponto de Fisher, situa-se em $(55 \%, \mathrm{R} \$ 2.600,99)$. Nesse ponto é indiferente a escolha do novo ou atual investimento, pois ambos apresentam o mesmo VPL a uma TMA de $55 \%$;

- Para $0 \% \leq \mathrm{TMA}<55 \%$ O VPL do atual investimento mostra-se superior ao VPL do novo investimento, portanto o primeiro deve ser escolhido;

- Para 55\% < TMA < 71\% o VPL do novo investimento apresenta-se ligeiramente maior do que o VPL do atual investimento, nesse caso deve ser escolhido; 
- Para uma TMA > 71\% ambos investimentos não são viáveis economicamente, pois apresentam VPL negativo.

\subsection{Análise dos Resultados}

Após o uso dos métodos de análise de investimento, observou-se que o novo investimento é insatisfatório para os objetivos da empresa, pois o VPL do atual em um maior intervalo de variação da TMA foi superior em comparação ao VPL do novo investimento.

Portanto, apesar de ambos investimentos não apresentarem inviabilidade do ponto de vista econômico-financeiro, o atual investimento se mostra mais viável em comparação a nova linha de brinquedos.

\section{Considerações Finais}

O presente estudo consistiu em analisar e comparar de modo econômico-financeiro os investimentos, baseando-se no fluxo de caixa, e utilizando as ferramentas do VPL e da TIR.

Ao longo do estudo, percebeu-se que para descobrir o melhor investimento seria necessário realizar uma análise mais detalhada por meio do método do ponto de Fisher. Por esse procedimento, foi possível observar a partir de um fluxo de caixa incremental, do valor presente líquido e da taxa interna de retorno, em quais momentos cada investimento se torna viável a partir da taxa mínima de atratividade. Ao final do estudo verificou-se por meio da metodologia utilizada, que o atual investimento continua sendo mais atrativo para a organização, abrindo a possibilidade de analisar outras linhas de produtos que sejam mais vantajosas que a atual.

Algumas das limitações encontradas para ser possível a análise desse estudo foi a pouca quantidade de conteúdo sobre o método do ponto de Fisher, de modo que inviabilizou uma abordagem mais aprofundada sobre a análise desse investimento. Outra consideração foi a dificuldade para organizar as informações da empresa no Demonstrativo do Resultado do Exercício (DRE) da organização. Muitas empresas não utilizam dessa estratégia, dificultando o planejamento financeiro dela e o alcance dos resultados desejados.

Desse modo, espera-se que este estudo possa viabilizar a essa e a outras organizações mais estratégias de viabilidade econômica financeira para a tomada de decisões de 
investimentos, visto que muitas organizações pouco utilizam dessas ferramentas que foram apresentadas e a dificuldade de encontrar conteúdos a respeito do método de Fisher.

\section{Referências}

ASSAF NETO, A. Finanças corporativas e valor. São Paulo: Atlas, 2005.

BRUNI, A. L. Avaliação de investimentos. São Paulo: Atlas, 2008.

FAMÁ, R, BRUNI LEAL, A. As decisões de investimentos com aplicação na HP12C e Excel. 3 ed. São Paulo: Atlas, 2012.

GITMAN, L. Princípios de administração financeira. 12a ed. São Paulo: Pearson, 2010

KASSAI, José Roberto. Conciliação entre a TIR e ROI: uma abordagem matemática e contábil do retorno do investimento. Cadernos de Estudos, São Paulo, FIPECAFI, n. 14., jul./dez. 1996.

MARTINS, E. Contabilidade vs. fluxo de caixa. Caderno de estudos da FIPECAFI. São Paulo: EDUSP, 1990.

SAMANEZ, Carlos Patrício. Engenharia econômica. São Paulo: Pearson Prentice Hall, 2009

SEBRAE - SERVIÇO BRASILEIRO DE APOIO ÀS MICRO E PEQUENAS EMPRESAS.

Empreendedorismo no Brasil relatório executivo (2014). Disponível

em:<https://m.sebrae.com.br/Sebrae/Portal\%20Sebrae/Estudos\%20e\%20Pesquisas/gem\%202014_relat

\%C3\%B3rio\%20executivo.pdf >. Acesso em: 01/11/2019

SOUZA, Alceu; CLEMENTE, Ademir. Decisões Financeiras e Análise de Investimento. São Paulo: Atlas, 2004..

SVIECH, V.; MANTOVAN, E.A. ANÁLISE DE INVESTIMENTOS: CONTROVÉRSIAS NA UTILIZAÇÃO DA TIR E VPL NA COMPARAÇÃO DE PROJETOS. Revista UniCuritiba 495, [S. 1.], p. 3-14, 2013. Disponível em:

http://revista.unicuritiba.edu.br/index.php/percurso/article/viewFile/657/495. Acesso em: 12 nov. 2019.

TABOSA, C.M.; RODRIGUES, M.V.; PINHEIRO, G.R. Analise de Viabilidade EconômicoFinanceira de um Empreendimento Imobiliário. XXXII ENCONTRO NACIONAL DE

ENGENHARIA DE PRODUÇÃO, Bento Gonçalves, p. 8-9, 15 out. 2012. 